\title{
Proof of Sun's conjecture on the divisibility of certain binomial sums
}

\author{
Victor J. W. Guo* \\ Department of Mathematics \\ East China Normal University \\ Shanghai 200062, People's Republic of China \\ jwguo@math.ecnu.edu.cn, http://math.ecnu.edu.cn/ ${ }^{\sim}$ jwguo \\ Submitted: Jan 28, 2013; Accepted: Oct 22, 2013; Published: Nov 22, 2013 \\ Mathematics Subject Classifications: 11B65, 05A10, 11A07
}

\begin{abstract}
In this paper, we prove the following result conjectured by Z.-W. Sun:

$$
(2 n-1)\left(\begin{array}{c}
3 n \\
n
\end{array}\right) \mid \sum_{k=0}^{n}\left(\begin{array}{c}
6 k \\
3 k
\end{array}\right)\left(\begin{array}{c}
3 k \\
k
\end{array}\right)\left(\begin{array}{c}
6(n-k) \\
3(n-k)
\end{array}\right)\left(\begin{array}{c}
3(n-k) \\
n-k
\end{array}\right)
$$

by showing that the left-hand side divides each summand on the right-hand side.

Keywords: congruences, binomial coefficients, super Catalan numbers, Stirling's
\end{abstract} formula

\section{Introduction}

In [4], Z.-W. Sun proved some new series for $1 / \pi$ as well as related congruences on sums of binomial coefficients, such as

$$
\sum_{n=0}^{\infty} \frac{n}{864^{n}} \sum_{k=0}^{n}\left(\begin{array}{c}
6 k \\
3 k
\end{array}\right)\left(\begin{array}{c}
3 k \\
k
\end{array}\right)\left(\begin{array}{c}
6(n-k) \\
3(n-k)
\end{array}\right)\left(\begin{array}{c}
3(n-k) \\
n-k
\end{array}\right)=\frac{1}{\pi}
$$

and, for any prime $p>3$,

$$
\sum_{n=0}^{p-1} \frac{n}{864^{n}} \sum_{k=0}^{n}\left(\begin{array}{c}
6 k \\
3 k
\end{array}\right)\left(\begin{array}{c}
3 k \\
k
\end{array}\right)\left(\begin{array}{c}
6(n-k) \\
3(n-k)
\end{array}\right)\left(\begin{array}{c}
3(n-k) \\
n-k
\end{array}\right) \equiv 0 \quad\left(\bmod p^{2}\right) .
$$

Sun [4] also proposed many interesting related conjectures, one of which is

\footnotetext{
*Supported by the Fundamental Research Funds for the Central Universities.
} 
Conjecture 1.1 [4, Conjecture 4.2] For $n=0,1,2, \ldots$, define

$$
s_{n}:=\frac{1}{(2 n-1)\left(\begin{array}{c}
3 n \\
n
\end{array}\right)} \sum_{k=0}^{n}\left(\begin{array}{c}
6 k \\
3 k
\end{array}\right)\left(\begin{array}{c}
3 k \\
k
\end{array}\right)\left(\begin{array}{c}
6(n-k) \\
3(n-k)
\end{array}\right)\left(\begin{array}{c}
3(n-k) \\
n-k
\end{array}\right) .
$$

Then $s_{n} \in \mathbb{Z}$ for all $n$. Also,

$$
\lim _{n \rightarrow \infty} \sqrt[n]{s_{n}}=64
$$

Sun himself has proved that $s_{n} \equiv 0(\bmod 8)$ for $n \geqslant 1$ and $s_{p-1} \equiv\lfloor(p-1) / 6\rfloor(\bmod p)$ for any prime $p$. In this paper, we shall prove that Conjecture 1.1 is true by establishing the following two theorems.

Theorem 1.2 For $0 \leqslant k \leqslant n$, we have

$$
\frac{1}{(2 n-1)\left(\begin{array}{c}
3 n \\
n
\end{array}\right)}\left(\begin{array}{c}
6 k \\
3 k
\end{array}\right)\left(\begin{array}{c}
3 k \\
k
\end{array}\right)\left(\begin{array}{c}
6(n-k) \\
3(n-k)
\end{array}\right)\left(\begin{array}{c}
3(n-k) \\
n-k
\end{array}\right) \in \mathbb{Z} .
$$

Note that, in $[5,6]$, Sun proved many similar results on the divisibility of binomial coefficients.

Theorem 1.3 For $n \geqslant 1$ and $0 \leqslant k<n / 2$, we have

$$
\begin{aligned}
& \left(\begin{array}{c}
6 k \\
3 k
\end{array}\right)\left(\begin{array}{c}
3 k \\
k
\end{array}\right)\left(\begin{array}{c}
6(n-k) \\
3(n-k)
\end{array}\right)\left(\begin{array}{c}
3(n-k) \\
n-k
\end{array}\right) \\
& \geqslant\left(\begin{array}{c}
6 k+6 \\
3 k+3
\end{array}\right)\left(\begin{array}{c}
3 k+3 \\
k+1
\end{array}\right)\left(\begin{array}{c}
6(n-k-1) \\
3(n-k-1)
\end{array}\right)\left(\begin{array}{c}
3(n-k-1) \\
n-k-1
\end{array}\right),
\end{aligned}
$$

and hence

$$
\frac{2}{2 n-1}\left(\begin{array}{l}
6 n \\
3 n
\end{array}\right) \leqslant s_{n} \leqslant \frac{n+1}{2 n-1}\left(\begin{array}{l}
6 n \\
3 n
\end{array}\right) .
$$

It is easy to see that (1.1) follows from (1.2) and Stirling's formula

$$
n ! \sim \sqrt{2 \pi n}\left(\frac{n}{e}\right)^{n} .
$$

\section{Proof of Theorem 1.2}

We need the following two lemmas.

Lemma 2.1 Let $m, n \geqslant 1$ and $0 \leqslant k \leqslant n$. Then

$$
\left(\begin{array}{c}
m n \\
n
\end{array}\right) \mid\left(\begin{array}{c}
2 m k \\
m k
\end{array}\right)\left(\begin{array}{c}
m k \\
k
\end{array}\right)\left(\begin{array}{c}
2 m(n-k) \\
m(n-k)
\end{array}\right)\left(\begin{array}{c}
m(n-k) \\
n-k
\end{array}\right) .
$$


Proof. Observe that

$$
\begin{aligned}
& \left(\begin{array}{c}
2 m k \\
m k
\end{array}\right)\left(\begin{array}{c}
m k \\
k
\end{array}\right)\left(\begin{array}{c}
2 m(n-k) \\
m(n-k)
\end{array}\right)\left(\begin{array}{c}
m(n-k) \\
n-k
\end{array}\right) /\left(\begin{array}{c}
m n \\
n
\end{array}\right) \\
& =\frac{(2 m k) !(2 m n-2 m k) !}{(m k) !(m n-m k) !(m n) !}\left(\begin{array}{c}
(m-1) n \\
(m-1) k
\end{array}\right)\left(\begin{array}{l}
n \\
k
\end{array}\right) .
\end{aligned}
$$

The proof then follows from the fact that numbers of the form

$$
\frac{(2 a) !(2 b) !}{a ! b !(a+b) !}
$$

called the super Catalan numbers, are integers (see $[1,2,7]$ ).

Lemma 2.2 Let $0 \leqslant k \leqslant n$ be integers. Then

$$
(2 n-1) \mid \frac{(6 k) !(6 n-6 k) !(2 n) !}{(3 k) !(3 n-3 k) !(3 n) !(2 k) !(2 n-2 k) !},
$$

or equivalently,

$$
\frac{(6 k) !(6 n-6 k) !(2 n) !(2 n-2) !}{(3 k) !(3 n-3 k) !(3 n) !(2 k) !(2 n-2 k) !(2 n-1) !} \in \mathbb{Z} .
$$

Corollary 2.3 Let $n \geqslant 1$. Then $(2 n-1)$ divides $\left(\begin{array}{l}6 n \\ 3 n\end{array}\right)$.

For the $p$-adic order of $n$ !, there is a known formula

$$
\operatorname{ord}_{p} n !=\sum_{i=1}^{\infty}\left\lfloor\frac{n}{p^{i}}\right\rfloor
$$

where $\lfloor x\rfloor$ denotes the greatest integer less than or equal to a real number $x$. In order to prove Lemma 2.2, we first establish the following result.

Lemma 2.4 Let $m \geqslant 2$ and $0 \leqslant k \leqslant n$ be integers. Then

$$
\begin{aligned}
& \left\lfloor\frac{6 k}{m}\right\rfloor+\left\lfloor\frac{6 n-6 k}{m}\right\rfloor+\left\lfloor\frac{2 n}{m}\right\rfloor+\left\lfloor\frac{2 n-2}{m}\right\rfloor \\
& \geqslant\left\lfloor\frac{3 k}{m}\right\rfloor+\left\lfloor\frac{3 n-3 k}{m}\right\rfloor+\left\lfloor\frac{3 n}{m}\right\rfloor+\left\lfloor\frac{2 k}{m}\right\rfloor+\left\lfloor\frac{2 n-2 k}{m}\right\rfloor+\left\lfloor\frac{2 n-1}{m}\right\rfloor,
\end{aligned}
$$

unless $m=3, n \equiv 2(\bmod 3)$, and $k \not \equiv 1(\bmod 3)$.

Proof. For any real numbers $x$ and $y$, we have (see, for example, [3, Division 8, Problems 8 and 136])

$$
\begin{aligned}
\lfloor 2 x\rfloor+\lfloor 2 y\rfloor & \geqslant\lfloor x\rfloor+\lfloor y\rfloor+\lfloor x+y\rfloor, \\
\lfloor x+y\rfloor & \geqslant\lfloor x\rfloor+\lfloor y\rfloor .
\end{aligned}
$$


It follows that

$$
\left\lfloor\frac{6 k}{m}\right\rfloor+\left\lfloor\frac{6 n-6 k}{m}\right\rfloor+\left\lfloor\frac{2 n}{m}\right\rfloor \geqslant\left\lfloor\frac{3 k}{m}\right\rfloor+\left\lfloor\frac{3 n-3 k}{m}\right\rfloor+\left\lfloor\frac{3 n}{m}\right\rfloor+\left\lfloor\frac{2 k}{m}\right\rfloor+\left\lfloor\frac{2 n-2 k}{m}\right\rfloor .
$$

Now suppose that (2.3) does not hold. Then we must have

$$
\begin{aligned}
\left\lfloor\frac{6 k}{m}\right\rfloor+\left\lfloor\frac{6 n-6 k}{m}\right\rfloor & =\left\lfloor\frac{3 k}{m}\right\rfloor+\left\lfloor\frac{3 n-3 k}{m}\right\rfloor+\left\lfloor\frac{3 n}{m}\right\rfloor, \\
\left\lfloor\frac{2 n}{m}\right\rfloor & =\left\lfloor\frac{2 k}{m}\right\rfloor+\left\lfloor\frac{2 n-2 k}{m}\right\rfloor, \\
\left\lfloor\frac{2 n-2}{m}\right\rfloor & <\left\lfloor\frac{2 n-1}{m}\right\rfloor,
\end{aligned}
$$

and so, by (2.6), $m \mid 2 n-1$, then by (2.5), $m \mid 2 k$ or $m \mid 2 n-2 k$. If $m \mid 2 k$, then $m \mid k$ (since $m \mid 2 n-1$ means that $m$ is odd), the identity (2.4) implies that

$$
\left\lfloor\frac{6 n}{m}\right\rfloor=2\left\lfloor\frac{3 n}{m}\right\rfloor .
$$

Since $m \mid 2 n-1$, the identity (2.7) can be written as

$$
\frac{2 n-1}{m}+\left\lfloor\frac{3}{m}\right\rfloor=2\left\lfloor\frac{n+1}{m}\right\rfloor .
$$

If $m \geqslant 4$, then the left-hand side of $(2.8)$ equals $(2 n-1) / m$, while the right-hand side of (2.8) belongs to

$$
\left\{\frac{2 n+2}{m}, \frac{2 n}{m}, \frac{2 n-2}{m}, \ldots\right\},
$$

a contradiction! Therefore, $m \leqslant 3$. Since $m \geqslant 2$ is odd, we must have $m=3$. Hence, $n \equiv 2(\bmod 3)$ and $k \equiv 0(\bmod 3)$. Similarly, if $m \mid 2 n-2 k$, then we deduce that $m=3$, $n \equiv 2(\bmod 3)$ and $k \equiv 2(\bmod 3)$. This proves the lemma.

Proof of Lemma 2.2. For any prime $p \neq 3$, by (2.2) and (2.3), we have

$$
\begin{aligned}
& \operatorname{ord}_{p}(6 k) !+\operatorname{ord}_{p}(6 n-6 k) !+\operatorname{ord}_{p}(2 n) !+\operatorname{ord}_{p}(2 n-2) ! \\
& \geqslant \operatorname{ord}_{p}(3 k) !+\operatorname{ord}_{p}(3 n-3 k) !+\operatorname{ord}_{p}(3 n) !+\operatorname{ord}_{p}(2 k) !+\operatorname{ord}_{p}(2 n-2 k) !+\operatorname{ord}_{p}(2 n-1) ! .
\end{aligned}
$$

For $p=3$, since $\operatorname{ord}_{3}(3 j) !=j+\operatorname{ord}_{3} j$ !, the inequality (2.9) reduces to

$$
\operatorname{ord}_{3}(2 n) !+\operatorname{ord}_{3}(2 n-2) ! \geqslant \operatorname{ord}_{3} k !+\operatorname{ord}_{3}(n-k) !+\operatorname{ord}_{3} n !+\operatorname{ord}_{3}(2 n-1) ! .
$$

Noticing that

$$
\frac{(2 n) !(2 n-2) !}{k !(n-k) ! n !(2 n-1) !}=\frac{1}{2 n-1}\left(\begin{array}{c}
2 n \\
n
\end{array}\right)\left(\begin{array}{l}
n \\
k
\end{array}\right)=\left(4\left(\begin{array}{c}
2 n-2 \\
n-1
\end{array}\right)-\left(\begin{array}{c}
2 n \\
n
\end{array}\right)\right)\left(\begin{array}{l}
n \\
k
\end{array}\right) \in \mathbb{Z},
$$


the inequality (2.10) holds. Namely, the inequality (2.9) is true for $p=3$. This completes the proof.

Proof of Theorem 1.2. By (2.1), one sees that

$$
\begin{aligned}
& \frac{1}{(2 n-1)\left(\begin{array}{c}
3 n \\
n
\end{array}\right)}\left(\begin{array}{c}
6 k \\
3 k
\end{array}\right)\left(\begin{array}{c}
3 k \\
k
\end{array}\right)\left(\begin{array}{c}
6(n-k) \\
3(n-k)
\end{array}\right)\left(\begin{array}{c}
3(n-k) \\
n-k
\end{array}\right) \\
& =\frac{1}{2 n-1} \frac{(6 k) !(6 n-6 k) !}{(3 k) !(3 n-3 k) !(3 n) !}\left(\begin{array}{c}
2 n \\
2 k
\end{array}\right)\left(\begin{array}{l}
n \\
k
\end{array}\right),
\end{aligned}
$$

which is an integer divisible by $\left(\begin{array}{l}n \\ k\end{array}\right)$ in view of Lemma 2.2.

\section{Proof of Theorem 1.3}

Let

$$
A_{n, k}=\left(\begin{array}{c}
6 k \\
3 k
\end{array}\right)\left(\begin{array}{c}
3 k \\
k
\end{array}\right)\left(\begin{array}{c}
6(n-k) \\
3(n-k)
\end{array}\right)\left(\begin{array}{c}
3(n-k) \\
n-k
\end{array}\right) .
$$

Then, for $n \geqslant 1$ and $0 \leqslant k<n / 2$, we have

$$
\frac{A_{n, k}}{A_{n, k+1}}-1=\frac{\left(36 n k+31 n-36 k^{2}-36 k-5\right)(n-2 k-1)}{(6 k+5)(6 k+1)(n-k)^{2}} \geqslant 0,
$$

i.e., $A_{n, k} \geqslant A_{n, k+1}$. Since $A_{n, k}=A_{n, n-k}$, for $n \geqslant 1$, we have

$$
2\left(\begin{array}{c}
6 n \\
3 n
\end{array}\right)\left(\begin{array}{c}
3 n \\
n
\end{array}\right)=2 A_{n, 0} \leqslant \sum_{k=0}^{n} A_{n, k} \leqslant(n+1) A_{n, 0}=(n+1)\left(\begin{array}{c}
6 n \\
3 n
\end{array}\right)\left(\begin{array}{c}
3 n \\
n
\end{array}\right) .
$$

In other words, the inequality (1.2) holds.

\section{References}

[1] J. W. Bober, Factorial ratios, hypergeometric series, and a family of step functions, J. Lond. Math. Soc. 79 (2009), 422-444.

[2] I. Gessel, Super ballot numbers, J. Symbolic Comput. 14 (1992), 179-194.

[3] G. Pólya and G. Szegö, Problems and Theorems in Analysis, vol. II, Grundlehren Math. Wiss., vol. 216. Springer, Berlin, 1976.

[4] Z.-W. Sun, Some new series for $1 / \pi$ and related congruences, preprint, arXiv:1104.3856.

[5] Z.-W. Sun, Products and sums divisible by central binomial coefficients, Electron. J. Combin. 20(1) (2013), \#P9.

[6] Z.-W. Sun, On divisibility of binomial coefficients, J. Austral. Math. Soc. 93 (2012), 189-201.

[7] S. O. Warnaar and W. Zudilin, A q-rious positivity, Aequat. Math. 81 (2011), 177183. 